\title{
In vitro and in vivo targeting of bladder carcinoma with metformin in combination with cisplatin
}

\author{
DONG WANG and XIAOHOU WU
}

\begin{abstract}
Department of Urology, The First Affiliated Hospital of Chongqing Medical University, Chongqing 400016, P.R. China
\end{abstract}
Received August 15, 2014; Accepted April 14, 2015

DOI: $10.3892 / 01.2015 .3267$

\begin{abstract}
Bladder cancer is the ninth most common carcinoma worldwide, and improving the sensitivity of this cancer to chemotherapy is a current clinical challenge. Metformin is a potentially useful therapeutic agent for the treatment of certain types of cancer. In the present study, metformin and cisplatin (a first-line chemotherapeutic agent for the treatment of bladder cancer) were administered to T24 and BIU-87 bladder cancer cells lines alone or in combination, prior to undergoing MTT assay and fluorescence-activated cell sorting analysis to determine cell viability and cell cycle distribution, respectively. Western blotting was used to examine the expression of proteins associated with the AMP-activated protein kinase (AMPK) and mammalian target of rapamycin (mTOR) signaling pathways. In addition, a xenograft model was constructed to evaluate the antitumor efficacy of metformin and cisplatin treatment, alone or in combination. Immunohistochemistry was performed to detect the expression levels of proteins associated with xenograft growth and angiogenesis. Furthermore, western blotting was performed to observe the expression of proteins associated with the AKT/mTOR signaling pathway in the xenograft model. The results demonstrated that the treatment of T24 and BIU-87 cells with metformin or cisplatin resulted in decreased tumor cell proliferation. However, the joint application of metformin and cisplatin was significantly more effective than that of each compound alone $(\mathrm{P}<0.05)$. Similarly, cells more markedly accumulated in the sub-G1 phase following joint treatment with metformin and cisplatin, compared with metformin or cisplatin treatment alone. In addition, human cell cycle signaling pathway western blotting arrays were performed, which identified the marked downregulation of phosphorylated (p)-mTOR and unchanged expression of p-AMPK, AMPK and mTOR
\end{abstract}

Correspondence to: Professor Xiaohou $\mathrm{Wu}$, Department of Urology, The First Affiliated Hospital of Chongqing Medical University, 1 You Yi Road, Chongqing 400016, P.R. China

E-mail: 14246385@qq.com

Key words: T24 cells, BIU-87 cells, metformin, cisplatin, bladder cell carcinoma following combined treatment with cisplatin and metformin. Concurrently, combined use of metformin and cisplatin markedly inhibited the growth and angiogenesis of xenografts generated from BIU-87 cells. Immunohistochemical analysis revealed that downregulation of the expression of specific proteins associated with AMPK promoted xenograft growth and angiogenesis, while western blotting revealed inhibition of the AKT/mTOR signaling pathway in xenografts treated with metformin in combination with cisplatin. Overall, the results of the present study demonstrated that the concurrent administration of metformin and cisplatin may result in enhanced antitumor efficacy compared with that of one agent alone, thus, providing a potential novel therapeutic strategy for the treatment of bladder cell carcinoma.

\section{Introduction}

As the ninth most common carcinoma diagnosis worldwide, $>330,000$ new cases of bladder carcinoma are diagnosed and >130,000 mortalities occur annually (1). Upon initial diagnosis of bladder cancer, approximately two-thirds of cases are diagnosed as non-muscle invasive bladder cancer (NMIBC) and almost one-third as muscle-invasive bladder cancer (MIBC). Although radical cystectomy is the gold standard treatment strategy for patients with MIBC, $\sim 50 \%$ of patients diagnosed with MIBC have imperceptible metastases at the time of treatment of the primary tumor. Furthermore, almost one-quarter of patients that undergo radical cystectomy present with lymph node involvement at the time of surgery. Therefore, this gold standard treatment strategy only provides a five-year survival rate of $~ 50 \%$ (2). To improve these results, peri-operative chemotherapy was introduced in the 1980s. Further studies have indicated that treatment with cisplatin-based neoadjuvant chemotherapy may significantly enhance overall survival (3). However, as only $\sim 50 \%$ of patients with MIBC respond to cisplatin-based chemotherapy (3), the identification of novel therapeutic strategies for the treatment of bladder carcinoma is required.

Metformin is an anti-diabetic agent that is typically prescribed to treat type 2 diabetes; however, it has recently received attention as a potentially useful therapeutic agent for the treatment of certain types of cancer (4-7). Previous studies have demonstrated that the combination of metformin and typical chemotherapeutic agents may inhibit the proliferation of breast cancer cells (8). Furthermore, in animal 
experiments, metformin in combination with the chemotherapeutic agent doxorubicin exhibited significant efficacy in the extermination of cancer stem cells, compared with that of doxorubicin alone (9). However, whether metformin is able to increase the sensitivity of bladder cancer to the chemotherapeutic agent cisplatin, as well as its exact mechanism remains to be elucidated.

Therefore, the current study aimed to analyze the antitumor efficacy of metformin treatment in combination with cisplatin on bladder cancer cell lines and explore the potential mechanism underlying this effect.

\section{Materials and methods}

Cell lines, antibodies and reagents. Two human bladder cancer cell lines, T24 and BIU-87, were obtained from the Shanghai Cell Bank (Shanghai, China) and cultured with $10 \%$ fetal bovine serum in a $37^{\circ} \mathrm{C}$ humidified atmosphere containing $5 \% \mathrm{CO}_{2}$. Metformin, cisplatin, MTT and dimethyl sulfoxide (DMSO) were all obtained from Sigma-Aldrich (St. Louis, MO, USA). Metformin and cisplatin were dissolved in DMSO at a concentration of $25 \mathrm{mmol} / \mathrm{l}$, aliquoted and stored at $-20^{\circ} \mathrm{C}$. Rabbit anti-human polyclonal IgG antibodies against adenosine monophosphate-activated protein kinase [AMPK; AMPKa1/2 (H-300); catalog no. sc-25792], phosphorylated (p)-AMPK [p-AMPK $\alpha 1 / 2$ (Thr 172); catalog no. sc-33524], mammalian target of rapamycin (mTOR; H-266; catalog no. sc-8319), p-mTOR (Ser 2448; catalog no. sc-101738), AKT [Akt1/2/3 (H-136); catalog no. sc-8312], p-AKT [p-Akt1/2/3 (Ser 473); catalog no. sc-33437] and $\beta$-actin (N-21; catalog no. sc-130656) were obtained from Santa Cruz Biotechnology, Inc. (Dallas, TX, USA), and mouse anti-human monoclonal antibodies against cluster of differentiation 34 (CD34; clone QBEnd 10; catalog no. IR632/IS632) and Ki-67 (clone MIB-1; catalog no. IR626/ IS626) were purchased from Dako North America, Inc. (Carpinteria, CA, USA). Horseradish peroxidase-conjugated goat anti-mouse IgG (Affinipure; catalog no. SA00001-2) secondary antibodies were obtained from Proteintech (Wuhan, China). In addition, the SP immunohistochemistry kit was purchased from Beijing Zhongshan Golden Bridge Biotechnology Co., Ltd. (Beijing, China) and Matrigel was purchased from BD Biosciences (Mountain View, CA, USA).

Treatment strategy. On day 1, a total of 5,000 cells/well were plated and grown on 96-well plates prior undergoing the following four treatment protocols: Untreated control, metformin alone, cisplatin alone or metformin in combination with cisplatin. On day 2 , new medium containing DMSO, metformin alone, cisplatin alone or metformin in combination with cisplatin was added following removal of the culture medium. The cells were incubated at $37^{\circ} \mathrm{C}$ in an atmosphere of $5 \% \mathrm{CO}_{2}$. Subsequently, assays were performed on day 4.

Cell viability assay. The effect of cisplatin and metformin on cell viability was analyzed by performing an MTT assay. Briefly, T24 or BIU-87 cells were subjected to the aforementioned treatment protocol with metformin $(0.5 \mu \mathrm{M})$ and/or cisplatin $(2 \mu \mathrm{m})$ for $0,24,36$ or $48 \mathrm{~h}$. Following removal of the medium and the addition of MTT solution, the cells were incubated for $1 \mathrm{~h}$. Subsequently, the MTT solution was replaced with $100 \mu 1$ DMSO. Thereafter, the optical density (OD) of the cells was measured using an $\mathrm{xMark}^{\mathrm{TM}}$ Microplate Absorbance Spectrophotometer (Bio-Rad Laboratories, Inc., Hercules, CA, USA; catalog no. 168-1150) at a wavelength of $560 \mathrm{~nm}$. Cell viability (\%) was calculated using the following equation: $\mathrm{OD}_{\text {treated wells }} / \mathrm{OD}_{\text {control wells }} \times 100 \%$.

Cell cycle analysis. T24 and BIU-87 cells were treated as follows: Metformin $(0.5 \mu \mathrm{M})$, cisplatin $(1 \mu \mathrm{M})$, metformin and cisplatin or DMSO as a control for $48 \mathrm{~h}$. Following treatment, cells were collected by trypsin digestion, washed in phosphate-buffered saline (PBS) and incubated for $24 \mathrm{~h}$ in $70 \%$ ethanol. Cells were resuspended in PBS containing $10 \mu \mathrm{g} / \mathrm{ml}$ propidium iodide and incubated in the dark for $30 \mathrm{~min}$ at $4^{\circ} \mathrm{C}$. The DNA content of the cells was analyzed by fluorescence-activated cell sorting (FACS) using a FACScan II and CellQUEST software (Beckton Dickinson, Moutain View, CA, USA). All remaining experimental steps for FACS analysis were performed as previously described (10).

Western blot analysis. Cells were seeded in 96-well microtiter plates and subjected to the aforementioned treatments, prior to the preparation of cell lysates, followed by protein fractionation and transfer, performed as previously described (11). The following human reactive antibodies were used for western blot analysis: primary AMPK (1:100 dilution), anti-p-AMPK (1:1,000 dilution), anti-mTOR (1:2,000 dilution) and anti-p-mTOR (1:1,000 dilution) antibodies and corresponding horseradish peroxidase-conjugated goat antimouse IgG secondary antibodies, at dilutions of 1:2,000, $1: 4,000,1: 2,000$ and 1:5,000, respectively. Protein expression was then visualized using Luminol reagent (Santa Cruz Biotechnology, Inc.) and an Odyssey ${ }^{\circledR}$ scanner (LI-COR Biosciences, Lincoln, NE, USA).

Establishment and treatment of mouse xenograft models. In the present study, all animals were obtained from, and experiments authorized by, the Experimental Animal Center of Chongqing Medical University (Chongqing, China). A total of 32 four-week-old male athymic BALB/c nu/nu mice (mean weight, $24.2 \mathrm{~g}$ ) were kept in individually ventilated cage systems with sterilized bedding at $20-25^{\circ} \mathrm{C}$. The mice received sterlized food and acidified water daily and were exposed to $12 \mathrm{~h}$ light/dark cycles. BIU-87 cells $\left(2 \times 10^{7}\right)$ in $100 \mu \mathrm{l}$ RPMI-1640 and $200 \mu \mathrm{l}$ Matrigel were subcutaneously injected into the left hip of each mouse. Mice were administered with metformin and cisplatin for four weeks and tumor volumes were subsequently measured, as described previously (12-14).

Immunohistochemical analysis of xenograft tumors. Mice were sacrificed by dislocation of the cervical vertebra, and tumors were surgically excised, fixed in $10 \%$ formalin, embedded in paraffin and cut into $5-\mathrm{mm}$ thick sections for immunohistochemical staining. All staining steps were performed according to the manufacturer's instructions. The primary antibodies and incubation conditions were as follows: Mouse monoclonal anti-Ki-67 at a 1:500 dilution in ready-to-use form 
A

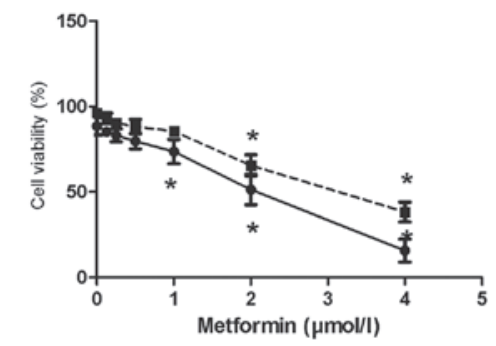

C

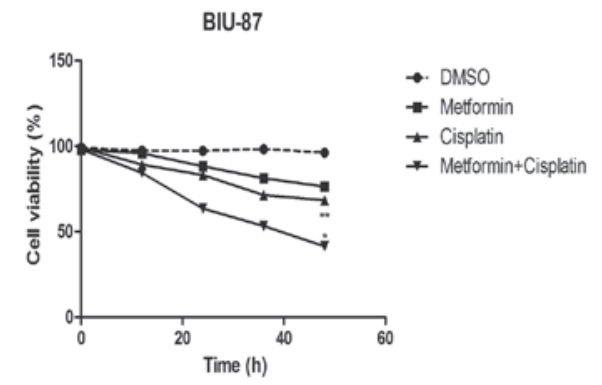

B

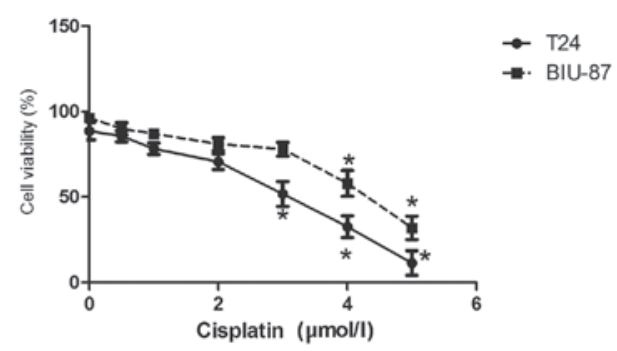

D

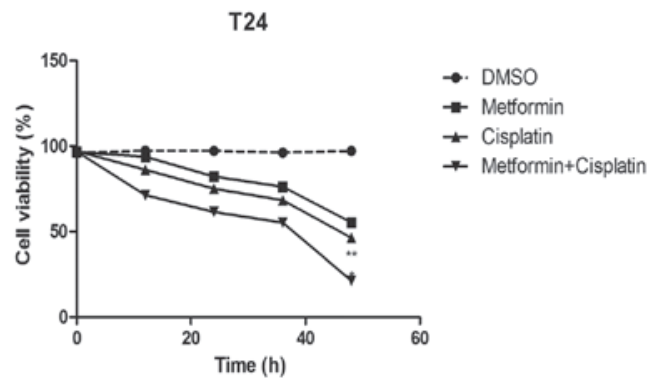

Figure 1. Metformin and cisplatin inhibit the growth of bladder cancer cell lines. Cells were treated with (A) metformin or (B) cisplatin at the indicated concentrations for $48 \mathrm{~h}$. (C) BIU-87 and (D) T24 cells were treated with metformin $(0.5 \mu \mathrm{M})$, cisplatin $(2 \mu \mathrm{M})$ or cisplatin in combination with metformin for 0 , 24,36 and $48 \mathrm{~h}$. Cell viability was determined by MTT assay. Data are presented as the mean \pm standard deviation of each group of cells from three independent experiments. ${ }^{*} \mathrm{P}<0.05$ vs. control group; ${ }^{* *} \mathrm{P}<0.05$ vs. cisplatin and metformin monotherapy groups.

A

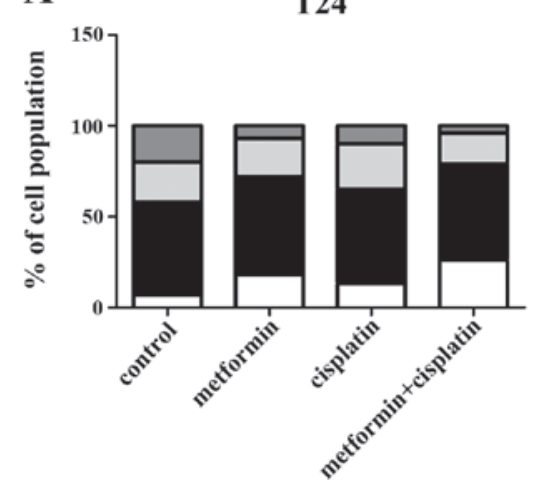

B

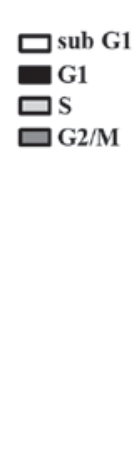

BIU-87

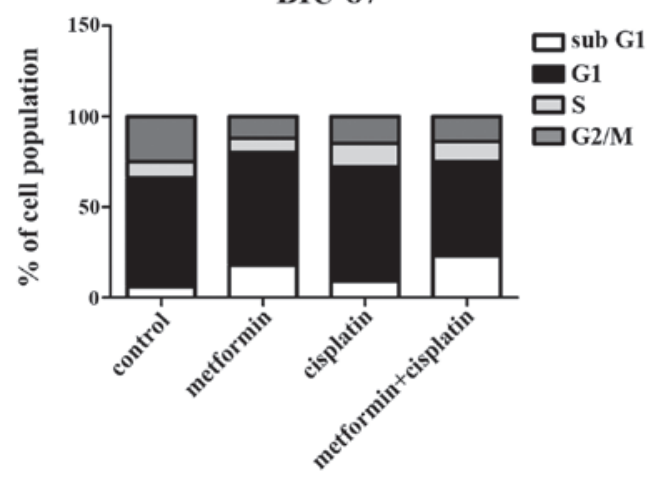

Figure 2. Cisplatin in combination with metformin alters bladder cancer cell cycle distribution. (A) T24 or (B) BIU-87 cells were treated for 48 h with metformin $(0.5 \mu \mathrm{M})$, cisplatin $(2 \mu \mathrm{M})$, metformin in combination with cisplatin or dimethyl sulfoxide (control). Cells were harvested and processed for cell cycle analysis. One of three similar experiments is indicated.

at room temperature for $30 \mathrm{~min}$, rabbit polyclonal anti-AKT in a 1:500 dilution at room temperature for $2 \mathrm{~h}$, rabbit polyclonal anti-p-AKT, in a 1:1,000 dilution at room temperature for $2 \mathrm{~h}$, and rabbit polyclonal anti-CD34 in a 1:1,500 dilution at $4^{\circ} \mathrm{C}$ overnight. The microvessel density (MVD) score was calculated as previously described (15).

Statistical analysis. Prism software (version 5.0; GraphPad Software, Inc., San Diego, CA, USA) was used to construct charts and perform all statistical analyses. Data were analyzed using the Student's $t$-test or one-way analysis of variance (Tukey's post test), as indicated in the figure legends, and are expressed as the mean \pm standard deviation. $\mathrm{P}<0.05$ was considered to indicate a statistically significant difference.

\section{Results}

Metformin and cisplatin inhibit T24 and BIU-87 cell viability in vitro. The MTT assay results demonstrated that a metformin concentration of $>1$ and $>2 \mu \mathrm{M}$ significantly inhibited T24 and BIU-87 cell viability, respectively (Fig. 1A). By contrast, a significant change in the viability of T24 and BIU-87 cells was induced by cisplatin at concentrations of 3 and $4 \mu \mathrm{M}$, respectively (Fig. 1B). Therefore, a concentration of $0.5 \mu \mathrm{M}$ metformin and $2 \mu \mathrm{M}$ cisplatin was selected for use in the subsequent experiments.

Combined treatment with metformin in combination with cisplatin inhibits bladder cancer cell proliferation. To observe the efficacy of metformin alone or in combination with cisplatin 
BIU-87
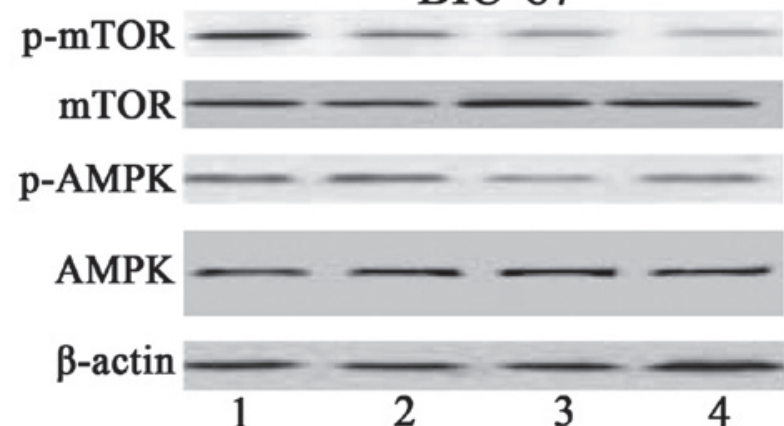

$\mathrm{T} 24$

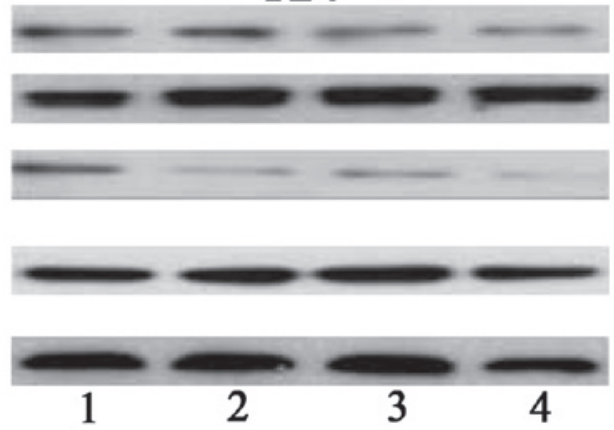

Figure 3. Metformin and cisplatin influence the protein expression levels in bladder cancer cells in vitro. Whole cell lysates were analyzed by western blotting using the indicated antibodies. The data exhibited are representative of three independent experiments. 1 , control group; 2 , cisplatin alone $(2 \mu \mathrm{M})$ treatment group; 3 , metformin alone $(0.5 \mu \mathrm{M})$ treatment group; 4 , metformin and cisplatin co-treatment group. mTOR, mammalian target of rapamycin; AMPK, adenosine monophosphate-activated protein kinase; p-, phosphorylated.
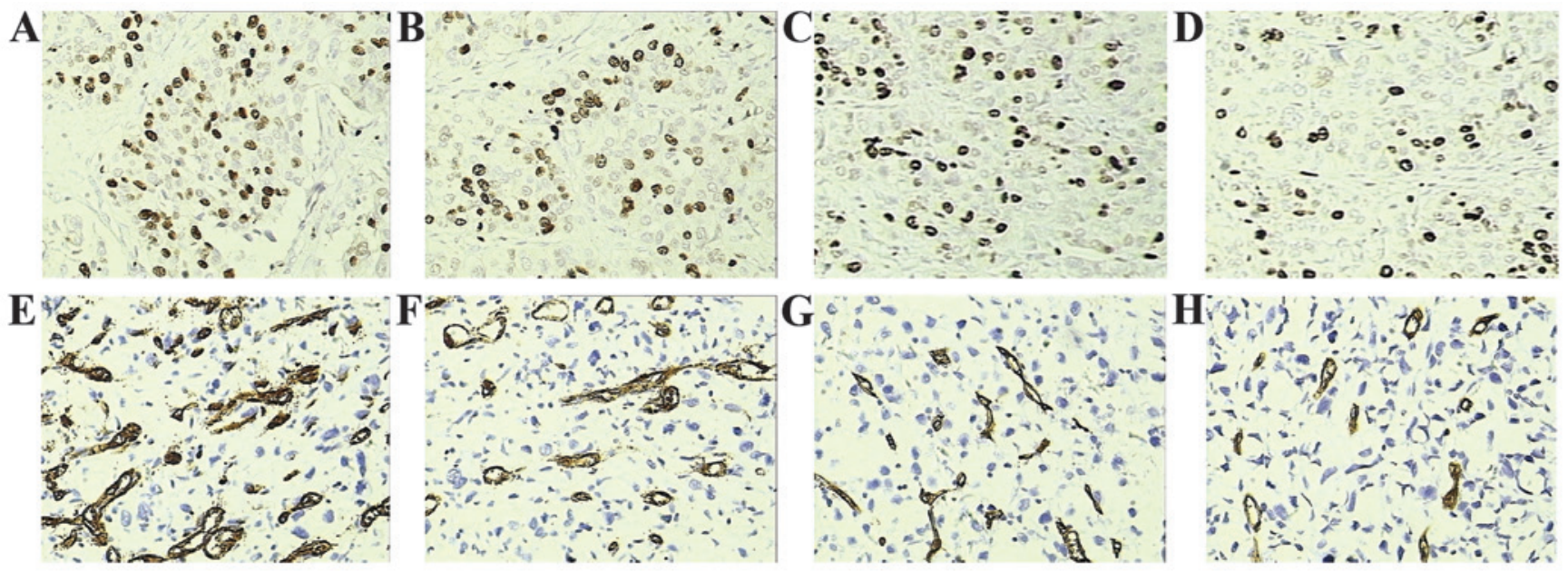

Figure 4. Cisplatin and metformin co-treatment significantly decreases tumor growth and angiogenesis. Immunolocalisation of (A-D) Ki-67 and (E-H) cluster of differentiation 34 in xenograft tumors derived from BIU-87 bladder carcinoma cells. (A and E) Control group; and groups treated with (B and F) cisplatin, (C and $\mathrm{G})$ metformin, and (D and $\mathrm{H}$ ) metformin and cisplatin (magnification, $\mathrm{x} 400$ ).

on the proliferation of T24 and BIU-87 cells, viable cells were treated with the indicated concentrations of the therapeutic agents for $0-48 \mathrm{~h}$ (Fig. 1C and D). The MTT assay results indicated that metformin in combination with cisplatin inhibited T24 and BIU-87 cell proliferation in a time-dependent manner. Furthermore, a significant decrease in the proliferation of T24 and BIU-87 cells was observed in the combined treatment group compared with that of the metformin and cisplatin alone treatment groups at $48 \mathrm{~h}(\mathrm{P}<0.05)$.

Combined treatment with metformin and cisplatin alters bladder cancer cell cycle distribution. Following the treatment of T24 and BIU-87 cells with metformin alone, cisplatin alone or cisplatin in combination with metformin for $48 \mathrm{~h}$, PI staining and FACS were used to analyze changes in the cell cycle in response to these agents. Cell cycle analysis identified that co-treatment resulted in an enhanced sub-G1 population when compared with monotherapy, for the two investigated cells types (Fig. 2). Thus, the current results indicated that the pro-apoptotic effect of metformin combined with cisplatin is greater than that of metformin and cisplatin treatment alone.
Cisplatin and metformin treatment alter the expression of proteins associated with the AMPK and mTOR signaling pathways in bladder cancer cell lines. Western blot analysis was performed to investigate the mechanism underlying the effects of metformin and cisplatin co-treatment on the cell cycle. In the T24 and BIU-87 cells, cisplatin-induced downregulation of p-mTOR was reinforced by co-treatment with metformin, while cisplatin-induced downregulation of p-AMPK was not altered by co-treatment with metformin. However, no marked changes in mTOR and AMPK expression were identified in the two types of cell (Fig. 3).

Cisplatin and metformin co-treatment decreases the size and weight of BIU-87 xenograft tumors. Considering that the aim of the present study was to establish a novel therapeutic strategy for the treatment of advanced bladder cancer cells, which are not sensitive to cisplatin, the BIU-87 cell line was selected for the in vivo investigation as its $50 \%$ inhibitory concentration following treatment with cisplatin for $48 \mathrm{~h}$ was significantly higher than that of T24 cells (data not shown). To assess the in vivo antitumor efficacy of co-treatment with cisplatin and metformin, nude mice bearing BIU-87 tumor xenografts were 
treated with cisplatin, metformin or a combination of the two agents for four weeks. The doses of cisplatin and metformin used were selected based on the results of initial investigations aimed at identifying the doses required to inhibit the growth of bladder cancer xenografts (data not shown). The size and weight of the tumor xenografts were significantly decreased following treatment with cisplatin or metformin alone compared with those of the untreated (control) xenografts (data not shown). Furthermore, the growth of combined cisplatin and metformin-treated xenografts was significantly inhibited when compared with monotherapy-treated xenografts. However, no significant body weight loss was observed during the four-week treatment process, indicating that the treatment strategies were well-tolerated with no obvious toxicity. Thus, the results of the present study indicate that the antitumor efficacy of cisplatin combined with metformin is greater than that of cisplatin and metformin alone.

Cisplatin in combination with metformin decreases tumor growth and angiogenesis. To investigate the tumor growth and angiogenesis mechanisms of cisplatin and metformin, immunostainings of Ki-67 and CD34 were performed, respectively (Fig. 4). The Ki-67 labeling index data revealed that tumors from mice treated with cisplatin without metformin had decreased levels of proliferation when compared with those of the control mice (Fig. 4A and B). Furthermore, combined treatment with metformin and cisplatin resulted in a marked decrease in the Ki-67 labeling index compared with that of cisplatin monotherapy (Fig. 4B and D). In agreement with the aforementioned data, MVD analysis revealed that mice treated with cisplatin or metformin alone exhibited decreased MVD compared with that of the control group (Fig. 4E-G). Similarly, a marked decrease in MVD was observed in the metformin and cisplatin co-treatment group compared with that of the cisplatin monotherapy group (Fig. 4A and $\mathrm{H}$ ).

Cisplatin and metformin co-treatment decreases the expression on $p$-AKT in BIU-87 xenografts. Finally, the metformin-induced inhibition of tumor growth and angiogenesis was analyzed by investigating the change in AKT protein expression levels associated with the AKT/mTOR signaling pathway. AKT expression was assessed by western blotting using equal weights of BIU-87 xenografts from nude mice. Expression of AKT protein was not significantly altered in the metformin-treated cells compared with that of the control cells. However, the expression levels of p-AKT protein were significantly decreased in the co-treatment group compared with those the monotherapy treatment groups $(\mathrm{P}<0.05)$ (data not shown).

\section{Discussion}

Human bladder cancer is one of the most fatal types of cancer in the world and, in certain countries, the incidence rate is increasing (1). In addition to surgical treatment, systematic chemotherapy is a common therapeutic strategy in the treatment of bladder cancer, particularly for patients with advanced and metastatic bladder cancer $(16,17)$. However, despite rapid shrinkage of the tumor mass following chemotherapy, the chemoresistance of carcinoma cells typically results in subsequent recurrence and metastasis of the cancer. For example, although a cisplatin-based combination chemotherapeutic strategy is a realistic alternative to cystectomy in advanced or metastatic bladder cancer, the development of cisplatin resistance is common amongst patients with bladder cancer (3). Recent studies have demonstrated that metformin exhibits an antiproliferative effect on carcinoma cells, directly as well as indirectly, by ameliorating insulin sensitivity, and decreasing hyperinsulinemia, respectively $(4,18,19)$. Therefore, the present study aimed to observe whether the application of cisplatin in combination with metformin exhibited greater efficacy compared with that of cisplatin monotherapy for the treatment of human bladder cancer.

In the present study, only a marginal decrease in the viability of cells treated with $2 \mu \mathrm{M}$ cisplatin or $0.5 \mu \mathrm{M}$ metformin alone was identified. However, cell viability was significantly reduced by co-treatment with cisplatin and metformin at these concentrations for $48 \mathrm{~h}$. These results indicated that combined treatment with cisplatin and metformin may be more effective against T24 and BIU-87 cell proliferation in vitro than treatment with cisplatin and metformin alone. Subsequently, the combined effect of cisplatin and metformin treatment was compared with that of the effect of cisplatin treatment alone. Cell cycle assays revealed an increased sub-G1 phase cell population in the cisplatin and metformin treatment groups in all cell lines. In particular, the ratio of sub-G1 cells was increased most potently by co-treatment compared with that of cisplatin and metformin monotherapy. The mechanism of this combined effect was then examined by measuring the expression levels of proteins associated with cellular AMPK and mTOR signaling. AMPK is an energy receptor within cells. Thus, a change in intercellular pressure or the consumption of glucose may increase the AMP/ adenosine triphosphate (ATP) ratio, activating AMPK expression by phosphorylation, and promoting the synthesis and utilization of glucose reabsorption. Therefore, AMPK is a critical regulatory pathway under normal physiological conditions (20). However, the predominant function of AMPK in tumors is to inhibit tumor cell proliferation and regulate apoptosis. It has been reported that metformin may induce a loss of mitochondrial membrane potential and inhibition of ATP production, which may consequently activate expression of the AMPK protein in prostate cancer cells $(21,22)$. The results of the present study demonstrated that treatment with cisplatin increased the expression of $\mathrm{p}$-AMPK; however, no significant change in the expression of p-AMPK was detected following treatment with metformin. Therefore, it appears that metformin does not inhibit the proliferation of bladder cancer cells via the AMPK signaling pathway. mTOR is a member of phosphatidyl inositol kinase-related enzyme family and is key in mediating the cell proliferation process (23). A previous study revealed that mTOR expression was increased in the majority cancer patients, and that hyperthyroidism and the phosphorylation of mTOR may promote tumor cell proliferation, differentiation, cell cycle regulation, growth and angiogenesis. However, mTOR expression may also be associated with the insensitivity to chemotherapy and targeted therapy of malignant tumors (24). To explore the mechanism by which metformin inhibits bladder cancer cells, the present study investigated whether $\mathrm{p}$-mTOR protein 
expression levels, which represent the degree of activation of the mTOR signaling pathway, were altered following treatment with cisplatin alone or in combination with metformin by western blot analysis. The present study verified the role of cisplatin in decreasing the transcriptional activity of mTOR and determined that cisplatin in combination with metformin exerted a more significant inhibitory effect on the mTOR signaling pathway.

Subsequently, whether cisplatin and metformin exhibited a combined effect on the xenograft tumor was examined. It was identified that the volume and weight of the xenograft tumor were significantly reduced by co-treatment with cisplatin and metformin, compared with the control and monotherapy groups. Furthermore, there was no apparent loss of body weight in the mice co-treated with cisplatin and metformin. Considering these results, it appears that combination therapy with cisplatin and metformin may respresent a safe and effective strategy for the inhibition of BIU-87 cell proliferation in vivo. To investigate the mechanisms underlying this combination effect, the protein expression levels of Ki-67 and CD34 were determined in isolated xenografts using immunohistochemical analysis. Ki-67 protein is typically considered to be a cell proliferation activity biomarker and research tool, with decreased Ki-67 protein expression indicating a general decline in cell proliferation (25). Thus, the present study assessed the Ki-67 labeling index and MVD, and clarified that combination therapy significantly inhibited tumor growth and angiogenesis compared with monotherapy. AKT is a major signaling molecule involved in regulating cell survival, proliferation, growth and angiogenesis. Furthermore, mTOR is a key substrate of AKT, with AKT able to activate mTOR and its downstream signaling pathways by directly phosphorylating serine sites on mTOR (26). In the present study, expression of p-AKT, the activated form of AKT, was significantly decreased in xenograft tumors following treatment with combined therapy of cisplatin and metformin, compared with that of the monotherapy group. This data further indicated that metformin enhances the cisplatin sensitivity of bladder cancer cells via the AKT/mTOR signaling pathway, as opposed to the AMPK signaling pathway.

The present study was limited by the use of only two types of bladder carcinoma cell and the generation of in vivo data from a xenograft tumor of a single cell line (BIU-87). Therefore, the evaluation of additional bladder cancer cell lines is required to establish the clinical potential of metformin and cisplatin co-administration for the treatment of bladder cancer.

In conclusion, the present study demonstrated that cisplatin combined with metformin had a synergistic anti-tumor effect in the treatment of human bladder carcinoma cells. In addition, the current study may encourage the future joint clinical application of metformin and cisplatin in bladder cancer. However, additional in vivo validation is required to determine whether the clinical application of this treatment strategy is able to achieve a satisfactory clinical outcome.

\section{Acknowledgements}

The authors would like to thank the Experimental Animal Center of Chongqing Medical University (Chongqing, China) for providing the animal research facility.

\section{References}

1. Ploeg M, Aben KK and Kiemeney LA: The present and future burden of urinary bladder cancer in the world. World J Urol 27: 289-293, 2009.

2. Stenzl A, Cowan NC, De Santis M, Kuczyk MA, Merseburger AS, Ribal MJ, Sherif A and Witjes JA; European Association of Urology: Treatment of muscle-invasive and metastatic bladder cancer: Update of the EAU guidelines. Actas Urol Esp 36: 449-460, 2012 (In Spanish).

3. Herr HW, Dotan Z, Donat SM and Bajorin DF: Defining optimal therapy for muscle invasive bladder cancer. J Urol 177: 437-443, 2007.

4. Bowker SL, Majumdar SR, Veugelers P and Johnson JA: Increased cancer-related mortality for patients with type 2 diabetes who use sulfonylureas or insulin: Response to Farooki and Schneider. Diabetes Care 29: 1990-1991, 2006.

5. Evans JM, Donnelly LA, Emslie-Smith AM, Alessi DR and Morris AD: Metformin and reduced risk of cancer in diabetic patients. BMJ 330: 1304-1305, 2005.

6. Ropelle ER, Pauli JR, Zecchin KG, Ueno M, de Souza CT, Morari J, Faria MC, Velloso LA, Saad MJ and Carvalheira JB: A central role for neuronal adenosine 5'-monophosphate-activated protein kinase in cancer-induced anorexia. Endocrinology 148: 5220-5229, 2007.

7. Franciosi M, Lucisano G, Lapice E, Strippoli GF, Pellegrini F and Nicolucci A: Metformin therapy and risk of cancer in patients with type 2 diabetes: Systematic review. PLoS One 8: e71583, 2013.

8. Liu B, Fan Z, Edgerton SM, Deng XS, Alimova IN, Lind SE and Thor AD: Metformin induces unique biological and molecular responses in triple negative breast cancer cells. Cell Cycle 8: 2031-2040, 2009.

9. Hirsch HA, Iliopoulos D, Tsichlis PN and Struhl K: Metformin selectively targets cancer stem cells, and acts together with chemotherapy to block tumor growth and prolong remission. Cancer Res 69: 7507-7511, 2009.

10. Abdelnour-Berchtold E, Cerantola Y, Roulin D, Dormond-Meuwly A, Demartines N and Dormond O: Rapamycin-mediated FOXO1 inactivation reduces the anticancer efficacy of rapamycin. Anticancer Res 30: 799-804, 2010.

11. Iwata H, Sato H, Suzuki R, Yamada R, Ichinomiya S, Yanagihara M, Okabe H, Sekine Y, Yano T and Ueno K: A demethylating agent enhances chemosensitivity to vinblastine in a xenograft model of renal cell carcinoma. Int J Oncol 38: 1653-1661, 2011.

12. Jensen MM, Jørgensen JT, Binderup T and Kjaer A: Tumor volume in subcutaneous mouse xenografts measured by microCT is more accurate and reproducible than determined by 18F-FDG-microPET or external caliper. BMC Med Imaging 8: $16,2008$.

13. Liu LZ, Zhou XD, Qian G, Shi X, Fang J and Jiang BH: AKT1 amplification regulates cisplatin resistance in human lung cancer cells through the mammalian target of rapamycin/p70S6K1 pathway. Cancer Res 67: 6325-6332, 2007.

14. Rocha GZ, Dias MM, Ropelle ER, et al: Metformin amplifies chemotherapy-induced AMPK activation and antitumoral growth. Clin Cancer Res 17: 3993-4005, 2011.

15. Miyake M, Anai S, Fujimoto K, et al: 5-fluorouracil enhances the antitumor effect of sorafenib and sunitinib in a xenograft model of human renal cell carcinoma. Oncol Lett 3: 1195-1202, 2012.

16. Juffs HG, Moore MJ and Tannock IF: The role of systemic chemotherapy in the management of muscle-invasive bladder cancer. Lancet Oncol 3: 738-747, 2002.

17. Gupta S and Mahipal A: Role of systemic chemotherapy in urothelial urinary bladder cancer. Cancer Control 20: 200-210, 2013

18. Fonseca EA, de Oliveira MA, Lobato NS, Akamine EH, Colquhoun A, de Carvalho MH, Zyngier SB and Fortes ZB.: Metformin reduces the stimulatory effect of obesity on in vivo Walker-256 tumor development and increases the area of tumor necrosis. Life Sci 88: 846-852, 2011.

19. Pollack MN: Insulin, insulin-like growth factors, insulin resistance, and neoplasia. Am J Clin Nutr 86: s820-s822, 2007.

20. Jalving M, Gietema JA, Lefrandt JD, de Jong S, Reyners AK, Gans RO and de Vries EG: Metformin: Taking away the candy for cancer? Eur J Cancer 46: 2369-2380, 2010. 
21. Kaaks R, Lukanova A and Kurzer MS: Obesity, endogenous hormones, and endometrial cancer risk: A synthetic review. Cancer Epidemiol Biomarkers Prev 11: 1531-1543, 2002.

22. Ben Sahra I, Laurent K, Giuliano S, Larbret F, Ponzio G, Gounon P, Le Marchand-Brustel Y, Giorgetti-Peraldi S, Cormont M, Bertolotto C, et al: Targeting cancer cell metabolism: The combination of metformin and 2-deoxyglucose induces p53-dependent apoptosis in prostate cancer cells. Cancer Res 70: 2465-2475, 2010.

23. Harris TE and Lawrence JC Jr: TOR signaling. Sci STKE 2003: re15, 2003

24. Zakikhani M, Blouin MJ, Piura E and Pollak MN: Metformin and rapamycin have distinct effects on the AKT pathway and proliferation in breast cancer cells. Breast Cancer Res Treat 123: 271-279, 2010.
25. Jiang XL, Zhang Y, Luo CL and Wu XH: Targeting renal cell carcinoma with gambogic acid in combination with sunitinib in vitro and in vivo. Asian Pac J Cancer Prev 13: 6463-6468, 2012.

26. Brazil DP and Hemmings BA: Ten years of protein kinase B signalling: A hard Akt to follow. Trends Biochem Sci 26: 657-664, 2001. 